\title{
Peribulbar injection of glucocorticoids for thyroid- associated ophthalmopathy and factors affecting therapeutic effectiveness: A retrospective cohort study of 386 cases
}

\author{
YUJIAO WANG, BAIXUE DU, MEI YANG, YANYAN ZHU and WEIMIN HE \\ Department of Ophthalmology, Ophthalmic Laboratory, West China Hospital, \\ Sichuan University, Chengdu, Sichuan 610041, P.R. China
}

Received September 27, 2019; Accepted May 7, 2020

DOI: $10.3892 /$ etm.2020.8896

\begin{abstract}
Thyroid-associated ophthalmopathy (TAO) is common in Graves' disease. However, to date, no standard treatment has been established for TAO. The present study aimed to assess peribulbar injection of corticosteroids for TAO treatment as well as factors affecting therapeutic effectiveness. A retrospective cohort study was performed at West China Hospital, Sichuan University (Chengdu, China). Patients with TAO were administered peribulbar injection of triamcinolone acetonide and dexamethasone monthly. Ocular signs after each injection were assessed until the end of treatment. All patients were followed up for at least six months. Best corrected visual acuity, proptosis values, eye motility assessed by the Hess chart, as well as eyelid width and downward movement were determined. In addition, clinical data were retrospectively reviewed to explore factors affecting therapeutic effectiveness by logistic regression analysis. In the present study, 386 patients with TAO (515 eyes) were evaluated; $71.37 \%$ of cases of eyelid swelling were relieved and upper eyelid retraction was improved in $47.58 \%$ of affected patients. Eye movement disorders, diplopia and strabismus were all alleviated to varying degrees, with few adverse reactions. Logistic regression analysis demonstrated that therapeutic effectiveness was relatively lower in males [odds ratio $(\mathrm{OR})=0.32, \mathrm{P}=0.001$ ] and patients with thyroid dysfunction $(\mathrm{OR}=0.41, \mathrm{P}=0.002)$, and that non-smokers had a higher odds of substantial improvement $(\mathrm{OR}=4.62, \mathrm{P}=0.008)$.
\end{abstract}

Correspondence to: Professor Weimin He, Department of Ophthalmology, Ophthalmic Laboratory, West China Hospital, Sichuan University, 37 Guoxue Street, Chengdu, Sichuan 610041, P.R. China

E-mail: weimin_he@yeah.net

Abbreviations: TAO, thyroid-associated ophthalmopathy; BCVA, best-corrected visual acuity; TA, triamcinolone acetonide; DEX, dexamethasone; CAS, clinical activity score

Key words: thyroid-associated ophthalmopathy, peribulbar injection, triamcinolone acetonide, dexamethasone, Graves' disease
The duration of TAO was not significantly associated with the clinical outcome. Patients with reduced disease severity and elevated clinical activity score exhibited higher effectiveness (all $\mathrm{P}<0.05$ ). In conclusion, peribulbar injection of corticosteroids is effective in treating mild to moderate TAO, with the therapeutic response affected by gender, smoking and disease severity.

\section{Introduction}

Thyroid-associated ophthalmopathy (TAO), also referred to as Graves' ophthalmopathy, is a chronic autoimmune orbital disease whose annual incidence rate approximates 16 females and 3 males per 100,000 individuals of the population in the United States (1). TAO represents the most common cause of orbital disease in adults; it is associated with thyroid dysfunction and constitutes the most common extrathyroidal sign of Graves' disease, featuring hyperthyroidism, diffuse goiter, ophthalmopathy, and, rarely, dermopathy (2).

Given its unclear pathogenesis and complex clinical manifestations, current treatment options for TAO, including glucocorticoids, radiotherapy and immunosuppressive agents, are not standardized, and their therapeutic effects remain largely elusive (3). This is particularly the case for TAO treatment at the inflammatory and unstable stage. At present, glucocorticoids are widely used to improve ocular symptoms by initiating anti-inflammatory responses (4).

The efficacy of glucocorticoids may be affected by several factors, particularly the administration route. For instance, systemically administered glucocorticoids may have multiple complications and side effects (5). However, retrobulbar or subconjunctival injection of corticosteroids for TAO treatment may avoid systemic complications (6). Previous studies have revealed the benefits of periocular injection of methylprednisolone and triamcinolone in improving the symptoms of TAO $(6,7)$.

At present, studies evaluating the effectiveness of various corticosteroids used locally to treat Chinese patients with TAO are scarce. Therefore, the present study aimed to retrospectively analyze the clinical outcomes of periorbital injection of triamcinolone acetonide (TA) combined with dexamethasone (DEX) in 386 Chinese patients with TAO and also assess factors that affect therapeutic effectiveness. 


\section{Patients and methods}

Study design and subjects. Patients diagnosed with $\mathrm{TAO}$ at the Ophthalmology Department of West China Hospital, Sichuan University (Chengdu, China) between November 2015 and August 2018 were enrolled in this retrospective cohort study. The inclusion criteria were as follows: i) First diagnosis of TAO; ii) clinical activity score (CAS) $\geq 3$ (active TAO). The following exclusion criteria were applied: i) Contraindication to steroids (diabetes or systemic hypertension, gastritis, psychosis or pregnancy); ii) previous treatment of TAO with steroids or radiation. Patients were included regardless of their endocrine status.

This retrospective study was performed according to the Declaration of Helsinki, and approved by the review board of West China Hospital, Sichuan University (Chengdu, China). Patients provided written informed consent for the publication of the images and data in this retrospective study.

Periorbital injection, clinical assessment and follow-up. Patients included in the present study were treated with injections of TA $(40 \mathrm{mg})$ combined with DEX $(2.5 \mathrm{mg})$ divided equally for injection to the supra- and infra-orbital foramen at 4-week intervals (monthly) until no further symptom improvement was observed. The dose of TA was determined referring to previous studies (6). The patient was placed in the supine position and sterile local anesthetic drops were placed in the eye. Subsequently, the eyelids were cleaned with povidone-iodine solution. Mixed corticosteroids were injected into the interior lateral and superior inner quadrants of the orbit, vertically and slowly by the same physician using a 26 -gauge disposable needle, avoiding the eyeball and surrounding vessels. The eye was closed, with light pressure immediately placed on the periorbital area for 10-15 min after needle withdrawal in order to spread the steroids and prevent hematoma formation. The patient's pulse and ocular condition were also observed.

All patients were followed up and assessed one month after each injection. At each visit, the following parameters were evaluated by the same ophthalmologist $(8,9)$ : Best-corrected visual acuity (BCVA; logMAR), eyelid width (mm), downward movement of the lids (mm), proptosis ( $\mathrm{mm}$; measured by a Hertel exophthalmometer), clinical activity score of TAO (CAS) $(9,10)$, modified NOSPECS class $(6,11)$, assessment of TAO severity (Table I) (12), limited eye motility (defined as $\geq 5^{\circ}$ reduction of eye movement in any direction assessed by the Hess chart) (13), intraocular pressure (IOP) and fundus features. Ocular complications linked to glucocorticoid injection and systemic adverse reactions were recorded. All patients underwent a monthly follow-up for half a year, followed by quarterly follow-up for at least another year.

Criteria for evaluating treatment effectiveness. Treatment effectiveness was defined as follows $(12,14)$ : a) Improvement in major outcome measures: Improvement in downward lid movement $\geq 2 \mathrm{~mm}$; amelioration in eye motility ( $\geq 5^{\circ}$ increase in eye movement in any direction); reduction in proptosis $\geq 2 \mathrm{~mm}$ (measured by Hertel exophthalmometer); b) improvement in auxiliary outcome measures: Reduction in eyelid width $\geq 2 \mathrm{~mm}$; mitigation of eyelid edema, conjunctival hyperemia and chemosis; c) Improvement in subjective symptoms.
Substantial improvement (very good effectiveness) was defined as changes in all major and auxiliary outcome measures and improvement in subjective symptoms. Minor improvement (good effectiveness) was defined as changes in one or more major and/or auxiliary outcome measures irrespective of subjective symptom changes. No change was defined as no improvement in any outcome measure after periorbital injection.

Statistical analysis. All data were analyzed with SPSS 22 (IBM Corp.). Continuous variables with a normal distribution were expressed as the mean \pm standard deviation and assessed by a paired t-test. Variables with a skewed distribution were tested by the Wilcoxon signed-rank test or the Kruskal-Wallis $\mathrm{H}$ test. Fisher's exact test or the $\chi^{2}$ test was used to compare categorical variables. The significance level was adjusted to $\alpha^{\prime}=0.017$ or $\alpha^{\prime}=0.0125$, considering multiple comparisons. Bivariate analysis was performed by the non-parametric Mann-Whitney U-test or Kruskal-Wallis test followed by Dunn's test to examine the associations of effectiveness with predictive factors.

In the present study, the ordinal logistic regression model conforming to the proportional odds assumption $(\mathrm{P}=0.29)$ was employed to evaluate the odds ratios (ORs) of factors potentially affecting the effectiveness of periorbital injection of corticosteroids. Accordingly, ordinal response variables (the overall effectiveness of periorbital injection) were defined as follows: i) 0 , 'no change'; ii) 1 , 'good' efficacy (minor improvement); and iii) 2, 'very good' efficacy (substantial improvement). The ORs between 'no change' vs. 'good'/'very good' efficacy and 'no change'/'good' vs. 'very good' efficacy were assumed to be identical (15-18). The relevant independent variables were as follows: Demographic characteristics, including gender and age; lifestyle parameters such as smoking and alcohol consumption; a thyroid function-associated factor, i.e., treatment response of thyroid dysfunction; and TAO-associated predictors, including CAS score, NOSPECS class and course of TAO. Variable selection was based on a previous study assessing the potential of variables for predicting the clinical outcome (10). A two-tailed $\mathrm{P}<0.05$ was considered to indicate statistical significance.

\section{Results}

Characteristics of the study population. A total of 386 patients (515 eyes) were included. There were 146 males (37.8\%) and 240 females (62.2\%), aged between 11 and 80 years (mean, $45.14 \pm 12.77$ years). The average duration of ocular signs and TAO symptoms was $14.72 \pm 6.63$ months (range, $8.5-21$ months). A total of 262 patients (67.9\%) presented with hyperthyroidism, including 89 cases of unilateral and 173 cases of bilateral TAO; 89 individuals (23.0\%) had euthyroid, including 53 unilateral and 36 bilateral cases $(\mathrm{P}>0.05)$. Furthermore, 35 cases (9.1\%) presented with primary hypothyroidism. Among the 222 patients treated with anti-thyroid drug, 98 recovered to have normal thyroid function. Of the 40 cases receiving ${ }^{131}$ I therapy, $32(80 \%)$ had hypothyroid. Those patients with thyroid dysfunction were advised to receive medical endocrinology treatment during the therapy for TAO.

According to the severity grades of NOSPECS, there were 173 cases of TAO (45.33\%) defined as grade 2, $79(20.47 \%)$ as 
Table I. Assessment of the severity of thyroid-associated ophthalmopathy.

Parameter

\begin{tabular}{lccc}
\cline { 2 - 4 } Severity & $\begin{array}{c}\text { Proptosis }^{\mathrm{a}} \\
(\mathrm{mm})\end{array}$ & Diplopia $^{\mathrm{b}}$ & $\begin{array}{c}\text { Visual acuity } \\
(\operatorname{logMAR})\end{array}$ \\
\hline Mild & $15-17$ & Intermittent & Normal \\
Moderate & $18-20$ & Inconstant & $0.09-0.3$ \\
Marked $^{\mathrm{c}}$ & $>20$ & Constant & $>0.3$ \\
\hline
\end{tabular}

aProptosis was evaluated by averaging three exophthalmometer readings. The upper normal limit was determined to be $15 \mathrm{~mm}$ in oriental populations (10). ${ }^{b}$ Diplopia: Intermittent, present only when fatigued; inconstant, present in secondary positions of gaze; constant, present in primary and reading positions. ${ }^{c}$ Marked: At least one pronounced, two moderate, or one moderate and two mild manifestations.

grade 3, $96(24.87 \%)$ as grade 4 and $38(9.84 \%)$ as grade 5. A total of 41 (34.25\%) of the 123 patients (including 118 males) with a smoking history ranging from 2 to 40 years successfully quit smoking during the treatment. All patients had varying degrees of eyelid swelling, retraction and sluggishness. Furthermore, 38 patients suffered from corneal epithelial defects without optic nerve involvement. The clinicopathological characteristics of the patients are summarized in Table II.

Therapeutic evaluation of periorbital injection. Fig. 1 provides images of three representative cases prior to and after treatment.

Symptomatic improvement. A total of 386 patients received periorbital injection therapy with a 3-5-month follow-up period. Among the 156 patients who experienced tearing, photophobia and blurred vision, 127 (81.41\%) exhibited improvement to varying degrees; the BCVA was significantly improved after treatment $(0.16 \pm 0.18$ vs. $0.35 \pm 0.12, \mathrm{P}=0.023)$. Pain due to eye movement was relieved in 31 of the 43 cases (72.09\%).

Sign improvement. Among the 276 cases with eyelid swelling, $225(81.52 \%)$ exhibited improvement; all conjunctival hyperemia cases were improved after treatment. Eyelid retraction was improved in 167 (62.08\%) of the 269 cases (representative cases showed in Fig. 1A and C). Upper eyelid sluggishness in 133 (46.02\%) of 289 patients showed improvement (Fig. 1B and D). A total of $49(41.52 \%)$ of 118 cases exhibited amelioration of proptosis (Fig. 1I and K). Eye motility disturbance was improved in $29(30.21 \%)$ of the 96 cases (Fig. 1J and L) and corneal epithelial involvement was alleviated in 9 (23.68\%) of the 38 cases affected.

Therapeutic effect profile. The patients received at least two injections and stopped when no significant effect was observed after the last injection. The number of patients exhibiting substantial improvement after 3 or 4 injections was higher than that of cases administered 2 injections $(\mathrm{P}<0.017)$. No significant difference in treatment effectiveness was observed between the 3 and 4 injection groups. These results indicated that the rate of patients with substantial improvement increased with the number of injections (Table II).

The patients were stratified into groups of mild to severe TAO based on the degree of proptosis, diplopia and visual acuity impairment, according to the assessment criteria of TAO severity (Table I). A total of 116 cases $(46.22 \%)$ of mild TAO were substantially improved, which was a higher rate compared with that in patients with moderate and severe TAO. Significantly more patients with moderate TAO had minor improvements after treatment compared with the severe TAO group (Table II).

Of the patients with TAO administered radioactive iodine ( $\left.{ }^{131} \mathrm{I}\right)$ treatment for Graves' hyperthyroidism, $80 \%$ presented with hypothyroid. Thus, thyroid function influenced the response to treatment. After periorbital injection of corticosteroids, significantly more patients in the primarily euthyroid group had 'very good' effectiveness in comparison with that in the abnormal after medical treatment (MT) group $(\mathrm{P}=0.008$; Table III). Compared with the group of primary euthyroid or patients with normal thyroid function after MT, the group of hypothyroidism after treatment with ${ }^{131}$ I therapy had a significantly lower proportion of patients with 'very good' effectiveness ( $\mathrm{P}=0.001$ and $\mathrm{P}=0.006$, respectively; Table III).

Management of adverse effects of periorbital injection. A total of 5 subjects developed subcutaneous ecchymosis, which was relieved gradually within two weeks during follow-up by pressure manipulation using swabs to hemostasis. Furthermore, 3 cases exhibited conjunctival edema that was eased after applying swabs with erythromycin ointment to return the prolapsed conjunctiva back into the eyelid. In addition, 1 case developed a self-limiting subconjunctival hemorrhage. A total of 31 cases $(8.03 \%)$ exhibited high IOP $(24.45 \pm 2.06 \mathrm{mmHg})$ after injection. After stopping the injections and administering ocular hypotensive agents, 3 cases had no improvement and 2 had increased fundus C/D values and visual field defects, as well as symptoms and signs of corticosteroid-induced glaucoma. Furthermore, 2 post-menopausal females ( 49 and 55 years old) had a menstruation after treatment. In addition, menstrual disorders occurred in 11 out of $90(16.67 \%)$ pre-menopausal females and the menstrual cycle became regular after stopping or completing the treatment. A total of 2 patients had moon face symptoms. No other ocular or systemic adverse reactions, including infection, penetrating eye, eye muscle motility disturbance (restriction of eye movement) and cerebral vascular events were observed (9). Furthermore, the rate at which patients experienced an effective outcome significantly increased with the number of periorbital injections.

Factors associated with the effectiveness of periorbital injection. According to the univariate analysis (Table II), clinical outcome was associated with patient gender, treatment outcomes of thyroid dysfunction (TOTD), duration of TAO, smoking habit, number of injections, CAS and disease severity (NOSPECS; P $<0.05$ ). However, age and drinking habits were not significantly associated with the effectiveness of periorbital injection.

According to the multivariate analyses, the adjusted factors that significantly and favorably associated with treatment effi- 

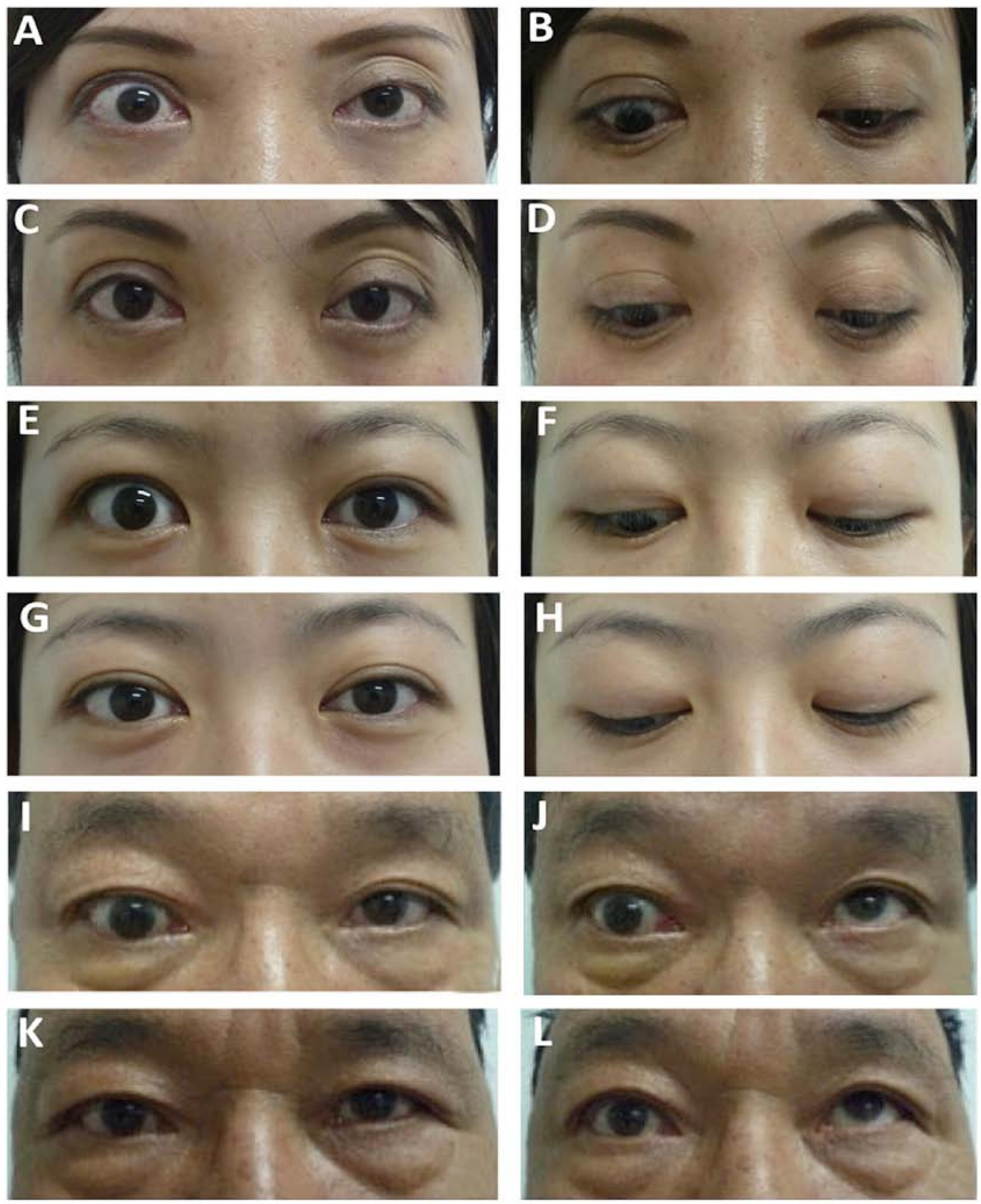

Figure 1. Thyroid-associated ophthalmopathy signs in patients are improved after treatment. (A-D) Female patient, 23 years old; (A and B) the right eye with (A) upper eyelid retraction and (B) drooping eyelid prior to treatment. (C) No eyelid retraction and (D) no drooping after periorbital injection of triamcinolone acetonide and dexamethasone. (E-H) Female patient, 36 years old; (E) right eye with upper eyelid swelling, eyelid retraction and conjunctival edema prior to treatment; (F) drooping eyelid prior to treatment; normal eyelid when looking forward (G) and looking downward (H) after periorbital injection. (I-L) Male patient, 51 years old; (I) right eye with upper eyelid contracture, with the Hirschberg test revealing the eye position $\mathrm{L} / \mathrm{R}=15^{\circ}$; (J) limited right-eye movement when looking upward; (K) normal eye position with no eyelid retraction after periorbital injection; (L) right eye movement was improved when looking upward after injection.

cacy in the ordinal logistic regression were female (vs. male) gender, no smoking, stable thyroid function, low NOSPECS class and high CAS (Table IV). Specifically, male patients were less likely to exhibit a substantial improvement compared with females $(\mathrm{OR}=0.32, \mathrm{P}=0.001)$. Compared with patients with a history of smoking, non-smokers had a higher odds of good effectiveness $(\mathrm{OR}=4.62, \mathrm{P}=0.008)$. Although patients with ocular sign for $\leq 1$ year had a higher odds of effective treatment than those with a longer disease course, the course of
TAO had no significant association with the clinical outcome $(\mathrm{P}=0.22)$. In terms of NOSPECS grades, patients with a grade of $\leq 3$ had a higher odds of substantial improvement compared with those with grade 5 (all $\mathrm{P}<0.05$ ). Patients with grade 4 tended to have a higher effectiveness than those with grade 5 , but with no statistical significance $(\mathrm{P}>0.05)$. Finally, patients with a relapse of thyroid dysfunction had poorer therapeutic effectiveness compared with those with primarily euthyroid or stable thyroid function (OR=0.41, $\mathrm{P}=0.002)$. 
Table II. Treatment effectiveness according to patient characteristics.

\begin{tabular}{|c|c|c|c|c|c|}
\hline \multirow[b]{2}{*}{ Variable } & \multirow[b]{2}{*}{ Cases (n) } & \multicolumn{3}{|c|}{ Effectiveness, n (\%) } & \multirow[b]{2}{*}{ P-value } \\
\hline & & Very good & Good & No change & \\
\hline Gender & & & & & $0.001^{\mathrm{a}}$ \\
\hline Male & 146 & $41(28.08)$ & $63(43.15)$ & $42(28.76)$ & \\
\hline Female & 240 & $135(56.25)$ & $82(34.17)$ & $23(9.60)$ & \\
\hline Age (years) & & & & & $0.150^{\mathrm{b}}$ \\
\hline $15-30$ & 21 & $12(57.14)$ & $7(33.33)$ & $2(9.52)$ & \\
\hline $31-45$ & 96 & $55(57.29)$ & $29(30.21)$ & $12(12.50)$ & \\
\hline $46-60$ & 152 & $64(42.11)$ & $56(38.84)$ & $32(21.05)$ & \\
\hline$\geq 61$ & 117 & $46(39.32)$ & $52(44.43)$ & $19(16.24)$ & \\
\hline TOTD & & & & & $0.001^{\mathrm{a}}$ \\
\hline $\mathrm{R} / \mathrm{E}$ & 212 & $129(60.85)$ & $63(29.72)$ & $20(9.43)$ & \\
\hline R/No & 174 & $53(30.46)$ & $76(43.68)$ & $45(25.86)$ & \\
\hline TAO duration & & & & & $0.001^{\mathrm{a}}$ \\
\hline$<1$ year & 284 & $145(51.06)$ & $102(35.92)$ & $37(13.03)$ & \\
\hline$\geq 1$ year & 102 & 31 (30.39) & $43(42.16)$ & $28(27.45)$ & \\
\hline Smoking & & & & & $0.001^{\mathrm{a}}$ \\
\hline Yes & 58 & $9(15.52)$ & $16(27.59)$ & $33(56.90)$ & \\
\hline No & 328 & $171(52.13)$ & $125(38.11)$ & $32(9.76)$ & \\
\hline Alcohol use & & & & & $0.080^{\mathrm{a}}$ \\
\hline Yes & 109 & $49(44.96)$ & $36(33.03)$ & $24(22.02)$ & \\
\hline No & 277 & $123(44.40)$ & $113(40.79)$ & $41(14.80)$ & \\
\hline CAS scores & & & & & $0.001^{\mathrm{b}}$ \\
\hline 3 & 135 & $36(26.67)$ & $58(42.96)$ & $41(30.37)$ & \\
\hline 4 & 130 & 54 (41.54) & $59(45.38)$ & $17(13.08)$ & \\
\hline 5 & 121 & $92(76.03)$ & $22(18.18)$ & $7(5.79)$ & \\
\hline NOSPECS class & & & & & $0.001^{\mathrm{b}}$ \\
\hline 2 & 173 & $119(68.79)$ & $48(27.75)$ & $6(3.47)$ & \\
\hline 3 & 79 & $42(53.16)$ & 29(36.71) & $8(10.13)$ & \\
\hline 4 & 96 & $29(30.21)$ & $36(37.50)$ & $31(32.29)$ & \\
\hline 5 & 38 & $5(13.16)$ & $13(34.21)$ & $20(52.63)$ & \\
\hline Number of injections & & & & & $0.02^{\mathrm{b}}$ \\
\hline 2 & 135 & $27(20.00)$ & $43(31.85)$ & $65(48.15)$ & \\
\hline 3 & 160 & $97(60.62)^{\mathrm{c}}$ & $63(39.38)$ & $0(0.00)$ & \\
\hline 4 & 91 & $56(61.54)^{\mathrm{d}}$ & $35(38.46)$ & $0(0.00)$ & \\
\hline Disease severity & & & & & $0.023^{\mathrm{b}}$ \\
\hline Mild & 251 & $116(46.22)$ & 87 (34.66) & 48 (19.12) & \\
\hline Moderate & 75 & $18(24.00)^{\mathrm{e}}$ & $36(48.00)$ & $21(28.00)$ & \\
\hline High & 60 & $14(23.33)^{\mathrm{f}}$ & $17(28.33)^{\mathrm{g}}$ & $29(48.33)$ & \\
\hline
\end{tabular}

${ }^{\mathrm{a}}$ Mann-Whitney U-test or ${ }^{\mathrm{b}}$ Kruskal-Wallis test was used for comparison among all groups of each variable. $\mathrm{P}<0.05$ was considered to indicate statistically significant difference. ${ }^{~} \mathrm{P}=0.017$ and ${ }^{\mathrm{d}} \mathrm{P}=0.01$ vs. 2 injections, 'very good' effectiveness, Kruskal-Wallis followed by Dunn's test. ${ }^{\mathrm{e}} \mathrm{P}=0.01$ and ${ }^{\mathrm{f}} \mathrm{P}=0.013$ vs. mild, 'very good' effectiveness, Kruskal-Wallis followed by Dunn's test. ${ }^{\mathrm{g}} \mathrm{P}=0.008$ vs. moderate, 'good' effectiveness. Disease severity classification was referred to the criterion of Table I. TOTD, treatment outcomes of thyroid dysfunction; R/E, Remission/ Euthyroid; R/No, Relapse/No response; TAO, thyroid-associated ophthalmopathy; CAS, clinical activity score.

\section{Discussion}

The present study demonstrated the effectiveness of periorbital injection of corticosteroids, namely TA and DEX in combination.
This clinical outcome was affected by several factors, including gender, smoking, as well as disease severity and activity.

Treatment options for different TAO stages remain controversial, as systemic administration of glucocorticoids may exert 
Table III. Effects of thyroid dysfunction on therapeutic effectiveness.

Effectiveness, n $(\%)$

\begin{tabular}{lccr} 
Thyroid function & Very Good & Good & No change \\
\cline { 3 - 4 } Primarily euthyroid $(\mathrm{n}=89)$ & $37(41.57)$ & $44(49.43)$ & $8(10.10)$ \\
Normal (after MT) $(\mathrm{n}=98)$ & $32(32.65)$ & $55(56.12)$ & $11(11.22)$ \\
Abnormal (after MT) $(\mathrm{n}=124)$ & $27(21.77)^{\mathrm{c}}$ & $67(54.03)$ & $30(24.19)$ \\
Hypothyroidism $\left(\right.$ after $\left.\mathrm{I}^{131}\right)(\mathrm{n}=32)$ & $5(15.62)^{\mathrm{a}, \mathrm{d}}$ & $11(34.38)^{\mathrm{b}}$ & $16(50.0)$
\end{tabular}

aP $=0.006$ vs. Normal (after MT), 'very good'. ' $\mathrm{P}=0.015$ vs. Normal (after MT), 'good'. 'P=0.008 vs. Primarily euthyroid, ('very good'). ${ }^{\mathrm{d}} \mathrm{P}=0.001$ vs. Primarily euthyroid ('very good'). MT, medical treatment.

Table IV. Results of ordinal logistic regression analysis regarding effective treatment.

\begin{tabular}{|c|c|c|c|c|}
\hline \multirow[b]{2}{*}{ Item } & \multirow[b]{2}{*}{ OR } & \multicolumn{2}{|c|}{$95 \% \mathrm{CI}$} & \multirow[b]{2}{*}{ P-value } \\
\hline & & Lower & Upper & \\
\hline Sex (male vs. female) & 0.32 & 0.16 & 0.62 & 0.001 \\
\hline TOTD (R/E vs. R/No) & 2.43 & 1.39 & 4.17 & 0.002 \\
\hline Smoking (yes vs. no) & 0.22 & 0.07 & 0.67 & 0.008 \\
\hline TAO duration ( $>1$ vs. $\leq 1$ year) & 0.72 & 0.42 & 1.21 & 0.220 \\
\hline \multicolumn{5}{|l|}{ CAS } \\
\hline 3 vs. 4 and 5 & 0.10 & 0.04 & 0.20 & $<0.001$ \\
\hline 3 and 4 vs. 5 & 0.20 & 0.09 & 0.42 & $<0.001$ \\
\hline \multicolumn{5}{|l|}{ NOSPECS class } \\
\hline 2 vs. 3,4 and 5 & 8.59 & 3.78 & 19.69 & $<0.001$ \\
\hline 2 and 3 vs. 4 and 5 & 4.06 & 1.52 & 10.80 & 0.005 \\
\hline 2,3 and 4 vs. 5 & 1.95 & 0.89 & 4.31 & 0.097 \\
\hline
\end{tabular}

Hosmer-Lemeshow goodness-of-fit test $\mathrm{P}=0.92$. 'no change', 'minor improvement' and 'substantial improvement' were defined as 0,1 and 2 , respectively. TOTD, treatment outcomes of thyroid dysfunction; TAO, severity; CAS, clinical activity score; OR, odds ratio; R/E, Remission/ Euthyroid; R/No, Relapse/No response.

numerous side effects (5). The present study demonstrated that periorbital injection of TA combined with DEX improved the symptoms of TAO with limited systemic adverse effects and side effects. TA, an insoluble long-acting synthetic fluorinated corticosteroid, is slowly absorbed into the eye; its potency is 5 times higher than that of cortisol, with effects sustained for 2-3 weeks or even longer (19). Thus, TA injection is required every 3-4 weeks. DEX is also a long-acting corticosteroid with a potency 25 times that of short-acting products (20), and combination with TA requires reduced injection dosages and times in patients with mild to moderate TAO compared with each corticosteroid applied as a monotherapy. According to our own experience, combination therapy of TA and DEX for patients with TAO may also achieve a prolonged local steroid concentration and higher potency to inhibit the inflammation compared with the use of TA only. In addition, a previous study indicated that periorbital injection of a combination of TA and DEX may improve lid retraction and limitation of eye movement in patients with TAO (21).
After periorbital injection, the symptoms of TAO exhibited various levels of improvement. Among TAO signs, substantial remissions in eyelid swelling, upper eyelid lag and retraction were achieved in the present study. Proptosis, involvement of eye muscles and corneal epithelial defects were also improved to varying degrees. Next, patients with active TAO were divided into groups based on disease severity (mild to severe) according to the degree of proptosis, diplopia and visual acuity impairment, which referred to the assessment criterion of TAO severity (13). This assessment can compensate for the subjective index of NOSPECS classification. Although substantial improvement was achieved in patients with mild to moderate proptosis, eye muscles were not successfully improved. These results indicated that periorbital injection of corticosteroids was more effective in patients with mild to moderate TAO.

The results also indicated that patients with TAO injected 3 or 4 times had a more pronounced improvement than those who received two injections. This suggests that the effec- 
tiveness of periorbital injection of these corticosteroids is cumulative.

Next, factors potentially predicting clinical outcome were assessed. The present analysis indicated that gender, smoking habits, CAS and NOSPECS class significantly affected the effectiveness of the treatment. Indeed, the current treatment was more effective in female patients compared with males, while smoking decreased the therapeutic effectiveness. A previous meta-analysis revealed a strong association of cigarette smoking with TAO progression and deterioration (22). In addition, smoking was previously reported to attenuate the effectiveness of systemic glucocorticoids as well as orbital radiotherapy (23). However, the pathogenic mechanisms underlying the effects of smoking in TAO remain to be fully elucidated. Thus, it is imperative for patients with TAO to quit smoking. The present study also demonstrated that patients with mild or moderate TAO were more likely to exhibit improvement after treatment compared with cases of severe TAO. These results indicated that periorbital injection of corticosteroids alone in cases of severe TAO may not suffice and should be combined with systemic administration of glucocorticoids or orbital radiotherapy. Furthermore, patients with a shorter course of TAO responded better to treatment than those with a longer course, although the duration of TAO was not significantly associated with the effectiveness of the treatment. Therefore, early treatment is also recommended for TAO. Previous studies indicated that progressive exacerbation of TAO characterized by lymphocyte infiltration may occur over 6-24 months and is considered the active progressive phase when anti-inflammatory therapy is indicated (24). This is followed by a weakened inflammatory activity and a static phase, which may lead to orbital tissue fibrosis. Cases of active TAO with high CAS scores were also likely to respond better to periorbital injection of steroids compared with the low CAS score group. The severity of TAO reflects the degree of orbital inflammation, which is remedied by the anti-inflammatory function of glucocorticoids (24). In addition, thyroid dysfunction may increase the risk of aggravation of TAO and reduce the effectiveness of periorbital injection. Previous studies revealed that thyroid-stimulatory hormone receptor antibody and thyroxine or triiodothyronine levels are associated with the worsening of TAO (25-27). However, further investigation is required to confirm the roles of the above factors in the development of TAO. It is known that ${ }^{131} \mathrm{I}$ administration triggers hypothyroidism and autoimmune inflammation, leading to the deterioration of TAO (28). The risk of radioactive iodine therapy is higher in comparison with that of anti-thyroid drugs $(29,30)$. As indicated above, patients with TAO and thyroid dysfunction had a poorer response to periorbital injection of corticosteroids than those with stable thyroid function. Therefore, thyroid function is considered the key factor influencing TAO treatment and ${ }^{131}$ I should be cautiously considered in hyperthyroid patients with ocular symptoms.

High intraocular pressure occurred in $8.03 \%$ of patients with complications caused by periorbital injection of corticosteroids and $83 \%$ of these patients recovered after discontinuation of treatment. A total of three patients had corticosteroid-induced glaucoma. A previous study demonstrated that local application of corticosteroids may lead to high IOP (31). While the associated pathogenesis remains elusive, a range of classical theories have been suggested. Steroid application has been previously found to reduce cellularity of the trabecular meshwork and increase extracellular matrix deposition, leading to increased aqueous outflow resistance and rise in IOP $(32,33)$. In the present study, after treatment, menstrual disorders arose in $16.67 \%$ of pre-menopausal patients, while menstruation occurred in two post-menopausal patients, whose condition returned to normal after treatment discontinuation. Menstrual irregularities and amenorrhea are known side effects of DEX $(34,35)$. Therefore, female patients should be informed of the possibility of menstrual disorders during treatment.

The present study had certain strengths, namely that the effectiveness of periocular injection of corticosteroids in the treatment of Chinese patients with TAO was assessed and factors affecting the clinical outcome were reported to guide clinical practice.

However, there were also certain limitations. First, selection bias could not be ruled out, as the recruited patients visited the hospital for ocular symptoms. Furthermore, the sample size was relatively small and should be increased in future investigations. These issues limit the generalizability of the present results. However, since West China Hospital (Chengdu, China), the largest hospital in Western China, encounters a large number of patients with TAO, some of whom have also come from another greater area. The present study may be considered to be representative of the population of patients with TAO in Southwest China.

In conclusion, the present study demonstrated that peribulbar injection of TA combined with DEX is an effective and safe treatment option for active TAO, particularly for mild to moderate active TAO. In addition, periorbital injection is a low-cost treatment and easy to administer, with almost no systemic side effects. However, patients with severe TAO may not achieve a satisfying response to periorbital injection of corticosteroids alone. Furthermore, in patients with TAO and thyroid dysfunction, appropriate endocrine therapy must be considered when applying periorbital injection of corticosteroids.

\section{Acknowledgements}

Not applicable.

\section{Funding}

The present study was supported by the 1.3.5 Project for Disciplines of Excellence-Clinical Research Incubation Project, West China Hospital, Sichuan University (grant no. 2018HXFH024) and the Post-Doctoral Research Project, West China Hospital, Sichuan University (grant no. 2019HXBH051) and Sichuan provincial science and technology program (18ZDYF1977).

\section{Availability of data and materials}

The datasets used and/or analyzed in the present study are available from the corresponding author on reasonable request. 


\section{Authors' contributions}

YJW and WMH designed the study. MY, YYZ and BXD performed experiments and recorded the data. YJW and MY were responsible for statistical analysis and data interpretation. YJW, BXD and WMH prepared the manuscript. All authors read and approved the final manuscript.

\section{Ethical approval and consent to participate}

All procedures performed in this retrospective study involving human participants were in accordance with and approved by the review board of West China Hospital of Sichuan University and conformed to the Declaration of Helsinki.

\section{Patient consent for publication}

Patients provided written informed consent for the publication of their images and data.

\section{Competing interests}

The authors declare that they have no competing interests.

\section{References}

1. Bahn RS: Graves' ophthalmopathy. N Engl J Med 362: 726-738, 2010.

2. Barrio-Barrio J, Sabater AL, Bonet-Farriol E, VelázquezVilloria Á and Galofré JC: Graves' ophthalmopathy: VISA versus EUGOGO classification, assessment, and management. J Ophthalmol 2015: 249125, 2015.

3. Bartalena L, Baldeschi L, Boboridis K, Eckstein A, Kahaly GJ, Marcocci C, Perros P, Salvi M and Wiersinga WM; European Group on Graves' Orbitopathy (EUGOGO): The 2016 European Thyroid Association/European Group on Graves' Orbitopathy Guidelines for the management of Graves' Orbitopathy. Eur Thyroid J 5: 9-26, 2016.

4. Dibas A and Yorio T: Glucocorticoid therapy and ocular hypertension. Eur J Pharmacol 787: 57-71, 2016.

5. Gao G, Dai J, Qian Y and Ma F: Meta-analysis of methylprednisolone pulse therapy for Graves' ophthalmopathy. Clin Exp Ophthalmol 42: 769-777, 2014.

6. Ebner R, Devoto MH, Weil D, Bordaberry M, Mir C, Martinez H, Bonelli $\mathrm{L}$ and Niepomniszcze $\mathrm{H}$ : Treatment of thyroid associated ophthalmopathy with periocular injections of triameinolone. $\mathrm{Br}$ J Ophthalmol 88: 1380-1386, 2004.

7. Alkawas AA, Hussein AM and Shahien EA: Orbital steroid injection versus oral steroid therapy in management of thyroidrelated ophthalmopathy. Clin Exp Ophthalmol 38: 692-697, 2010.

8. Frueh BR: Why the NO SPECS classification of Graves' eye disease should be abandoned, with suggestions for the characterization of this disease. Thyroid 2: 85-88, 1992.

9. Mourits MP, Koornneef L, Wiersinga WM, Prummel MF, Berghout $\mathrm{A}$ and van der Gaag R: Clinical criteria for the assessment of disease activity in Graves' ophthalmopathy: A novel approach. Br J Ophthalmol 73: 639-644, 1989.

10. Mourits MP, Prummel MF, Wiersinga WM and Koornneef L: Clinical activity score as a guide in the management of patient with Graves' ophthalmopathy. Clin Endocrinol 47: 9-14, 1997.

11. Wiersinga WM, Prummel MF, Mourits MP, Koornneef L and Buller HR: Classification of the eye changes of Graves' disease. Thyroid 1: 357-360, 1991.

12. Bartalena L, Pinchera A and Marcocci C: Management of Graves' Ophthalmopathy: Reality and perspectives. Endocr Rev 21: 168-199, 2000.
13. Watts P, Nayak H, Lim MK, Ashcroft A, Madfai HA and Palmer H: Validity and ease of use of a computerized Hess chart. J AAPOS 15: 451-454, 2011.

14. Nakamura T, Wakakura M and Ishikawa S: Exophthalmometric values in contemporary Japanese population. Jpn J Clin Ophthalmol 46: 1031-1035, 1992.

15. McCullagh P: Regression models for ordinal data. J R Stat Soc 42: 109-142, 1980

16. Ananth CV and Kleinbaum DG: Regression model for ordinal responses: A review of methods and applications. Int J Epidemiol 26: 1323-1332, 1997.

17. Bender R and Grouven U: Using binary logistic regression models for ordinal data with non-proportional odds. J Clin Epidemiol 51: 809-816, 1998.

18. Adeleke KA and Adepoju AA: Ordinal logistic regression model: An application to pregnancy outcomes. J Math Stat 6: 279-285, 2010.

19. Xu D, Liu Y, Xu H and Li H: Repeated triamcinolone acetonide injection in the treatment of upper lid retraction in patients with thyroid-associated ophthalmopathy. Can J Ophthalmol 47: 34-41, 2012.

20. Zoorob RJ and Cender D: A different look at corticosteroids. Am Fam Physician 58: 443-450, 1998.

21. Bagheri A, Abbaszadeh M and Yazdani S: Intraorbital steroid injection for active thyroid ophthalmopathy. J Ophthalmic Vis Res 15: 69-77, 2020.

22. Vestergaard P: Smoking and thyroid disorders-A meta-analysis. Eur J Endocrinol 146: 153-161, 2002.

23. Hegediüs L, Brix TH and Vestergaard P: Relationship between cigarette smoking and Graves' ophthalmopathy. J Endocrinol Invest 27: 265-271, 2004.

24. Hiromatsu Y, Eguchi H, Tani J, Masataka K and Yasuo T: Graves' Ophthalmopathy: Epidemiology and natural history. Intern Med 53: 353-360, 2014.

25. Wall JR and Lahooti H: Pathogenesis of thyroid eye diseasedoes autoimmunity against the TSH receptor explain all cases. Endokrynol Pol 61: 222-227, 2010.

26. Stan MN and Bahn RS: Risk factors for development or deterioration of graves' ophthalmopathy. Thyroid 20: 777-783, 2010.

27. Träisk F, Tallstedt L, Abraham-Nordling M, Andersson T, Berg G, Calissendorff J, Hallengren B, Hedner P, Lantz M, Nyström E, et al: Thyroid-associated ophthalmopathy after treatment for Graves' hyperthyroidism with antithyroid drugs or iodine-131. J Clin Endocrinol Metab 94: 3700-3707, 2009.

28. Bartalena L, Marcocci C, Bogazzi F, Manetti L, Tanda ML, Dell'Unto E, Bruno-Bossio G, Nardi M, Bartolomei MP, Lepri A, et al: Relation between therapy for hyperthyroidism and the course of Graves' ophthalmopathy. N Engl J Med 338: 73-78, 1998.

29. Wang R, Tan J, Zhang G, Zheng W and Li C: Risk factors of hepatic dysfunction in patients with Graves' hyperthyroidism and the efficacy of 131 iodine treatment. Medicine 96: e6035, 2017.

30. Louvet C, De Bellis A, Pereira B, Bournaud C, Kelly A, Maqdasy S, Roche B, Desbiez F, Borson-Chazot F, Tauveron I and Batisse-Lignier M: Time course of Graves' orbitopathy after total thyroidectomy and radioiodine therapy for thyroid cancer. Medicine (Baltimore) 95: e5474, 2016.

31. Jones R III and Rhee DJ: Corticosteroid-induced ocular hypertension and glaucoma: A brief review and update of the literature. Curr Opin Ophthalmol 17: 163-167, 2006.

32. Skuta GL and Morgan RK: Corticosteroid induced glaucoma. In: The glaucoma. Ritch R, Shields MB and Krupin T (eds). 2nd edition. Mosby, St. Louis, MO, pp17721188, 1996.

33. Razeghinejad MR and Katz LJ: Steroid-induced iatrogenic glaucoma. Ophthalmic Res 47: 66-80, 2012.

34. Mahajan VK Sharma NL, Sharma RC and Garg G: Twelve-year clinico-therapeutic experience in pemphigus: A retrospective study of 54 cases. Int J Dermatol 44: 821-827, 2005.

35. Abramavicius S, Velickiene D and Kadusevicius E: Methimazoleinduced liver injury overshadowed by methylprednisolone pulse therapy. Medicine 96: e8159, 2017.

This work is licensed under a Creative Commons Attribution-NonCommercial-NoDerivatives 4.0 International (CC BY-NC-ND 4.0) License. 